\title{
Limits of the Oberbeck-Boussinesq approximation in a tall differentially heated cavity filled with water
}

\author{
D. Kizildag ${ }^{\mathrm{a}}$, I. Rodriguez ${ }^{\mathrm{a}}$, A. Oliva ${ }^{\mathrm{a}, *}$, O. Lehmkuhl ${ }^{\mathrm{a}, \mathrm{b}}$ \\ ${ }^{a}$ Centre Tecnologic de Transferencia de Calor (CTTC) \\ Universitat Politecnica de Catalunya (UPC) \\ ETSEIAT, Colom 11, 08222, Terrassa, Barcelona, Spain \\ Fax: +34937398920 E-mail: cttc@cttc.upc.edu \\ ${ }^{b}$ Termo Fluids, S.L. \\ Av., Jacquard 97-E, 08222 Terrassa, Barcelona, Spain
}

\begin{abstract}
The present work assesses the limits of the Oberbeck-Boussinesq (OB) approximation for the resolution of turbulent fluid flow and heat transfer inside a tall differentially heated cavity of aspect ratio $\Gamma=6.67$ filled with water $\left(\operatorname{Pr}=3.27, R a=2.12 \times 10^{11}\right)$. The cavity models the integrated solar collector-storage element installed on an advanced façade. The implications of the Oberbeck-Boussinesq approximation is submitted to investigation by means of direct numerical simulations (DNS) carried out for a wide range of temperature differences. Non-Oberbeck-Boussinesq (NOB) effects are found to be relevant, specially for temperature differences $\Delta T>30^{\circ} \mathrm{C}$, in the accurate estimation of heat transfer, stratification, and transition to turbulence location.
\end{abstract}

Keywords: non-Oberbeck-Boussinesq effects, turbulent natural convection, differentially heated cavity

\footnotetext{
*Corresponding author; Tel:+34 9373981 92, e-mail: cttc@cttc.upc.edu (A. Oliva)
} 


\section{Introduction}

Natural convection flows in cavities have attracted the attention of many researchers due to their presence in many applications of industrial relevance, such as the air flow in buildings, heat transfer in solar collectors, nuclear reactors, among others. Substantial work has been devoted to two canonical configurations: i) a cavity heated from below; ii) a cavity heated from the sides. The first configuration is known as the Rayleigh-Bénard problem, where the gravitational vector is parallel to the thermal gradient. The latter case is commonly referred to as the differentially heated cavity (DHC) problem.

The present work focusses on the DHC configuration, which is characterized by a thermal gradient orthogonal to the gravitational field. Several contributions to understand the physics of this flow appeared in the literature along the last three decades. The vast majority of the presented work was related to air-filled cavities (Prandtl number $\operatorname{Pr} \sim 0.71$ ) and, considering the so-called Oberbeck-Boussinesq (OB) approximation. This approximation, being more mathematical than physical, considers constant thermophysical properties of the fluid, except for the density in the gravitational term of the momentum equation. By means of this approximation, the exact governing equations are modified to derive more tractable equations. As indicated in the work of Gray \& Giorgini [1], the employment of the OB approximation can be justified for a wide range of temperature differences, as the air-filled enclosures are concerned.

The earlier efforts to model this phenomena addressed mainly steady laminar two-dimensional flows [2]. Driven by the increased computational 
power, works on two- and three-dimensional transitional and turbulent flows gradually emerged in the last two decades, each time addressing more chaotic flows $[3,4,5]$. The accumulated knowledge shows that although the DHC configuration represents a simple geometry, the flow gets extremely complex for sufficiently large Rayleigh numbers $(R a)$, since it undergoes a gradual transition to a chaotic state beyond a critical $R a$ (see for instance Refs. [6, 7]). For high $R a$, different regimes are expected to coexist within the enclosure: i) laminar flow in the upstream part of the vertical boundary layer and ii) turbulent flow at some location downstream in the vertical boundary layer $[8,9]$. Furthermore, due to the flow regime established in the hot and cold walls, some level of stratification is expected at the midheight of the cavity. This stratification phenomenon taking place in the core has been studied for a wide range of aspect ratios [10]. There have been found controversies about the stratification measured experimentally and numerically, which can be attributed to thermal radiation effects [11]. For more details about flow configuration, scale analysis, and stability properties the reader is referred to Refs. $[12,13,14]$.

For the DHC configuration, works conducted without OB approximation are relatively limited in number. Paolucci et al. [15] investigated numerically the transition from laminar to turbulent flow in a two dimensional air filled cavity for different aspect ratios. Later Paolucci [16] studied the two-dimensional turbulent natural convection in an air filled square cavity for $R a=10^{10}$. Both studies were conducted without OB approximation, although the density variations in these works were restricted to the values within the so-called OB limit. Suslov and Paolucci [17] studied the linear 
stability of the natural convection flow in a tall DHC under non-OberbeckBoussinesq (NOB) conditions, investigating the dependence of the critical Rayleigh number on the temperature difference. Their results showed that the instabilities can be controlled by a competition between the shear and bouyancy mechanisms. Later, Mlaouah et al. [18] studied the case of a square air-filled cavity by means a low-Mach number approximation, indicating the difficulties in describing the transition behaviour of the flow at higher Rayleigh numbers if the exact equations are not used. Recently Szewc et al. [19] obtained steady laminar natural convection solutions for a square cavity of $R a=10^{5}$ by means of smoothed particle hydrodynamics method, comparing results with $\mathrm{OB}$ and general NOB formulations. They studied different sets of Gay-Lussac numbers including considerable departures from the socalled OB regime, pointing significant variations in velocity and temperature profiles with respect to the reference OB solution.

For the Rayleigh-Bénard configuration, several authors studied this flow without considering the OB approximation. These studies can serve as reference to understand the physics of the DHC problem, despite the expected differences due to the direction of the thermal gradient. The NOB effects in low Prandt gasses were studied by Robinson and Chan [20], and by Madruga and Riecke [21]. For the case of liquids, NOB effects on the temperature profile and the Nusselt number were previously studied for water by Ahlers et al. [22]. Later, Sugiyama et al. [23] extended their study to glycerol. In these cases NOB effects are mainly due to variations of the viscosity with temperature. However, Ahlers et al. [24] also studied NOB effects caused by the strong dependence of the thermal expansion coefficient on the temperature 
for ethane near its critical point.

The present work is focussed on a water-filled DHC configuration. When the working fluid is water, i.e. greater Prandtl numbers compared with air, obtaining solutions for the governing equations gets even more complicated since the boundary layer becomes thinner than for air at the same conditions. As a consequence, there is an increasing demand for excessively fine grids in space and time in order to capture the smallest scales of the turbulent flow. Additionally, in most applications using water under practical working conditions, the OB approximation has to be questioned. This approximation is valid if the fluid properties are weakly dependent on the temperature or the temperature difference within the walls are small enough. However, under real working conditions, important variations of thermopyhsical properties can be observed. In this study, we aim at addressing situations which are well out of the OB regime.

Due to the large computational resources required to compute threedimensional (3D) simulations, being even more demanding for water-filled cavities, this work considers two-dimensional (2D) simulations. This hypothesis is expected to be adequate to study the validity of the OB approximation, and the conclusions are expected to be acceptable without the loss of generality. In fact, there are several studies which support this hypothesis in the natural convection flow within enclosures. Schmalzl et al. [25] studied the Rayleigh-Bénard flow by means of 2D and 3D simulations, concluding that both show similar characteristics in the flow structure and global quantities at high Prandtl numbers, while differing -even qualitativelyat Prandtl numbers smaller than unity. DeLuca et al. [26] showed for the 
OB Rayleigh-Bénard convection that the most important flow features can be well captured by 2D simulations. More recently, Sugiyama et al. [27] also performed 2D simulations for the Rayleigh-Bénard convection considering both $\mathrm{OB}$ approximation and $\mathrm{NOB}$ effects, showing that 2D approach reflects well the dynamics of the boundary layer and the behaviour of the Nusselt numbers. Trias et al. [28] performed 2D and 3D simulations of a DHC of aspect ratio 4 for Ra up to $10^{10}$, observing that $2 \mathrm{D}$ computations were in good agreement with 3D ones in terms of time-averaged structures and averaged local and overall Nusselt numbers. It is important to note that they pointed out significant deviations in second order turbulent statistics. We are aware that especially in the turbulent regions, 2D approximation may not be suitable to predict the actual flow structures, however it must be borne in mind that the primary scope of this work is to shed more light on the NOB effects, studying qualitatively and quantitatively the influence of temperature difference when the conditions depart from the so-called OB regime. Under these circumstances, the 2D approximation can be considered less critical since NOB effects can be present in both 2D and 3D cases, and 2D simulations can be considered sufficient to capture the dynamics of the flow and particularly the influence of NOB effects.

This paper is organized as follows: in Section 2 we present the description of the case, the mathematical and numerical model. Section 3 is devoted to the validation of the numerical model. In Section 4, the numerical results are discussed, and finally Section 5 presents the concluding remarks. 


\section{Description of the case}

The integrated solar collector-storage element to model is a two-dimensional cavity (see Figure 1 ) of width $W=0.11 \mathrm{~m}$, with two vertical isothermal walls of height $H=0.735 \mathrm{~m}$. Wall temperatures are set to $T_{h}$ and $T_{c}$ so that $T_{m}=\left(T_{h}+T_{c}\right) / 2=52{ }^{\circ} \mathrm{C}$. The adopted geometry has an aspect ratio of $\Gamma=6.68$. The cavity has adiabatic end walls. No-slip boundary conditions are imposed at the walls, which are rigid and impermeable.

This work aims at studying the relevance of the NOB effects in the DHC filled with water and to shed light into the qualitative and quantitative implications of OB approximation. For this reason a wide range of temperature differences $\left(\Delta T=T_{h}-T_{c}=10,20,30,40,60\right)$ is chosen to compare both OB and NOB results obtained for the same mean Rayleigh and Prandtl numbers in order to shed light on qualitative and quantitative implications of NOB approximation.

In this paper, the OB case will be referred to as $B S Q 10$, while the reference to a particular NOB case will be done with the associated temperature difference, e.g. NOB10, NOB20, etc.

\section{Mathematical and numerical model}

In bouyancy driven flows a common approach is to consider constant thermophysical properties of the fluid, with the exception of the density variations that are only taken into account in the bouyancy forces, i.e., the so-called OB approximation. Thus, the temperature dependence of density in the bouyancy force is linearized as: 


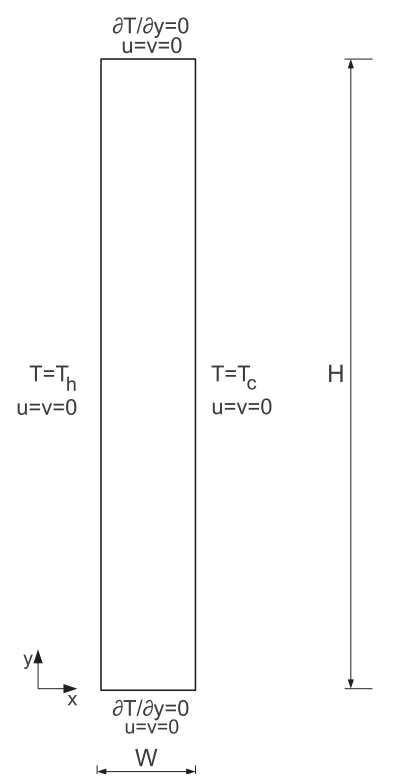

Figure 1: Geometry and boundary conditions

$$
\rho(T)=\rho_{m}-\rho_{m} \beta_{m}\left(\mathbf{T}-T_{m}\right)
$$

where $\rho_{m}$ is the density, $\beta_{m}$ is the thermal expansion coefficient $\left(\beta_{m}=\right.$ $\left.-\rho_{m}^{-1} \partial \rho /\left.\partial T\right|_{T_{m}}\right)$ at the mean temperature $T_{m}=\left(T_{h}+T_{c}\right) / 2$. These assumptions have several implications. First, continuity equation is treated in its incompressible form, neglecting acoustic phenomena, which in the case of liquids has no major implications. Furthermore, for liquids, deviations from the aforementioned hypothesis are mainly due to viscosity variations, as the viscosity strongly decreases with the temperature increase.

Assuming the $\mathrm{OB}$ approximation, the finite volume discretization of the incompressible Navier-Stokes and continuity equations on an arbitrary mesh can be written as: 


$$
\begin{aligned}
\mathbf{M u} & =\mathbf{0} \\
\Omega \frac{\partial \mathbf{u}}{\partial t}+\mathbf{C}(\mathbf{u}) \mathbf{u}+\nu \mathbf{D u}+\Omega \rho^{-1} \mathbf{G} p+f & =\mathbf{0} \\
\Omega \frac{\partial \mathbf{T}}{\partial t}+\mathbf{C}(\mathbf{u}) \mathbf{T}+\alpha \mathbf{D}(\mathbf{T}) & =\mathbf{0}
\end{aligned}
$$

where $\mathbf{u} \in \mathbb{R}^{3 n}, \mathbf{T} \in \mathbb{R}^{n}$ and $p \in \mathbb{R}^{n}$ are the velocity vector, temperature and pressure, respectively (here $\mathrm{n}$ applies for the total number of control volumes (CV) of the discretized domain), $\nu$ is the kinematic viscosity, $\alpha$ is the thermal diffusivity $\left(\alpha=k / \rho C_{p}\right), k$ is the conductivity, and $C_{p}$ is the specific heat. $f$ is the body force $f=\beta\left(T_{0}-T_{m}\right)$ g. Note that all the thermophysical properties are evaluated at $T_{m}$. Convective and diffusive operators in the momentum equation for the velocity field are given by $\mathbf{C}(\mathbf{u})=(\mathbf{u} \cdot \nabla) \in \mathbb{R}^{3 n \times 3 n}, \mathbf{D}=$ $\nabla^{2} \in \mathbb{R}^{3 n \times 3 n}$ respectively. Gradient and divergence (of a vector) operators are given by $\mathbf{G}=\nabla \in \mathbb{R}^{3 n \times 3 n}$ and $\mathbf{M}=\nabla \cdot \in \mathbb{R}^{n \times 3 n}$ respectively. $\Omega \in \mathbb{R}^{n}$ is a matrix with the volumes of the cells.

In our work when considering the NOB effects, the thermophysical properties of water are calculated following a temperature dependence:

$$
\phi(T)=\Sigma_{n=0}^{N} a_{n} T^{n}
$$

where $\phi$ is the thermophysical property $\left(\rho, C_{p}, \mu, k\right), T$ is the temperature in Kelvins, and the coefficients $a_{n}$ of the equation 5 are given in Table 3.

Thus, equations 2 to 4 with variable thermophysical properties of incompressible fluid read: 


\begin{tabular}{ccccc}
\hline $\mathrm{n}$ & $\rho$ & $C_{p}$ & $\mu$ & $k$ \\
& $\left(\mathrm{~kg} / \mathrm{m}^{3}\right)$ & $(\mathrm{J} / \mathrm{kg} \cdot \mathrm{K})$ & $(\mathrm{kg} / \mathrm{m} \cdot \mathrm{s})$ & $(\mathrm{W} / \mathrm{m})$ \\
\hline 0 & $8.487 \cdot 10^{2}$ & $2.282 \cdot 10^{4}$ & $1.942 \cdot 10^{-1}$ & $-2.978 \cdot 10^{0}$ \\
1 & $1.29 \cdot 10^{0}$ & $-1.94 \cdot 10^{2}$ & $-2.19 \cdot 10^{-3}$ & $3.07 \cdot 10^{-2}$ \\
2 & $-2.6 \cdot 10^{-3}$ & $7.52 \cdot 10^{-1}$ & $9.89 \cdot 10^{-6}$ & $-9.8 \cdot 10^{-5}$ \\
3 & & $-1.29 \cdot 10^{-3}$ & $-2.22 \cdot 10^{-8}$ & $1.43 \cdot 10^{-7}$ \\
4 & & $8.3 \cdot 10^{-7}$ & $2.4 \cdot 10^{-11}$ & $-8.2 \cdot 10^{-11}$ \\
5 & & & $-1.1 \cdot 10^{-14}$ & \\
\hline
\end{tabular}

Table 1: Coefficients $a_{n}$ of the equation 5 to calculate the temperature dependent thermophysical properties of water.

$$
\begin{aligned}
\mathbf{M u} & =\mathbf{0} \\
\Omega \rho(T) \frac{\partial \mathbf{u}}{\partial t}+\mathbf{C}(\rho(T) \mathbf{u}) \mathbf{u}+\mathbf{D}(\nu(T) \mathbf{u})+\Omega \mathbf{G} p-\rho(T) \mathbf{g} & =\mathbf{0} \\
\Omega \rho(T) C_{p}(T) \frac{\partial \mathbf{T}}{\partial t}+\mathbf{C}\left(C_{p}(T) \rho(T) \mathbf{u}\right) \mathbf{T}+\mathbf{D}(k(T) \mathbf{T}) & =\mathbf{0}
\end{aligned}
$$

Considering the reference scales for length, time, velocity, temperature and dynamic pressure as $L_{r e f}=H, t_{r e f}=\left(H^{2} / \alpha\right) R a^{-0.5}, v_{r e f}=(\alpha / H) R a^{0.5}$, $\Delta T_{\text {ref }}=T_{h}-T_{c}, p_{\text {ref }}=\rho\left(\alpha / H^{2}\right) R a$, respectively, OB thermal convection in the cavity is governed by the Rayleigh number $\left(R a=g \beta\left(T_{h}-T_{c}\right) H^{3} \operatorname{Pr} / \nu^{2}\right)$, Prandtl number $(\operatorname{Pr}=\nu / \alpha)$, and height aspect ratio $\Gamma=H / W$. The problem under study is defined by $\operatorname{Pr}=3.27, R a=2.12 \times 10^{11}$, and $\Gamma=6.67$ due to the particular geometry, mean temperature, and thermophysical properties of the present investigation.

The governing equations are discretized on a collocated unstructured grid arrangement by means of second-order conservative schemes [29]. Such dis- 
cretization preserves the symmetry properties of the continuous differential operators, i.e., the conservation properties are held if, the convective term is discretized by a skew-symmetric operator and the diffusive term is approximated by a symmetric, positive-definite coefficient matrix. These properties ensure both stability and conservation of the global kinetic-energy balance on any grid. Energy transport is also discretized by means of a conservative scheme.

For the temporal discretization of the momentum equation, a two-step linear explicit scheme on a fractional-step method has been used, while for the pressure-gradient term an explicit first-order scheme has been employed [30]. This methodology has been previously used with accurate results for solving the flow over bluff-bodies with massive separation (see for instance $[31,32])$, but also for the turbulent natural convection in enclosures [33, 34].

It might be argued that collocated meshes do not conserve kinetic energy when fractional step method is used due to the contribution of the pressure gradient term to the evolution of the kinetic energy $[35,36]$. However, pressure errors have been proven to be of the order of $\mathcal{O}\left(\triangle x^{2} \triangle t^{2}\right)$ [36]. Thus, in DNS the impact of such errors should be minimum due to the grid sizes and time-steps used.

\section{Validation of the numerical model}

The OB model is validated by means of a $2 \mathrm{D}$ differentially heated cavity case with $R a=10^{10}, \operatorname{Pr}=0.71$, and height aspect ratio $\Gamma=4$ for which Trias et al. [28] presented benchmark DNS results.

For validation purposes, the computational grid defined by Trias et al. 


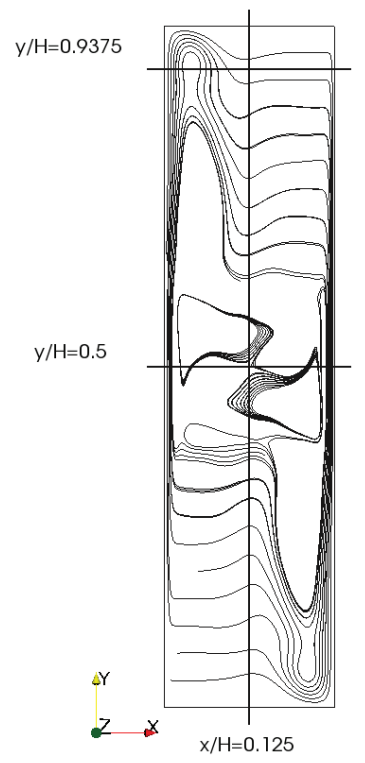

Figure 2: Locations in the cavity where computational results are compared.

[28] as $C 2 D$ is employed. The numerical results obtained with this computational grid are compared with the finer $C C 2 D$ results of the same authors. The comparison is carried out considering overall averaged Nusselt numbers, and first order statistics at different locations within the cavity.

The overall averaged Nusselt number calculated yielded 100.46, which is within a relative error of $0.14 \%$ with respect to the benchmark solution $(N u=$ 100.60), indicating a satisfactory agreement, that can also be confirmed when the first order flow variables are compared at different locations of the cavity (see Figure 2). As can be seen in Figure 3, the velocity and temperature profiles are well predicted, not only at the mid height of the cavity where the flow is laminar, but also at the top $(y / H=9.9375)$ where the flow is turbulent. 


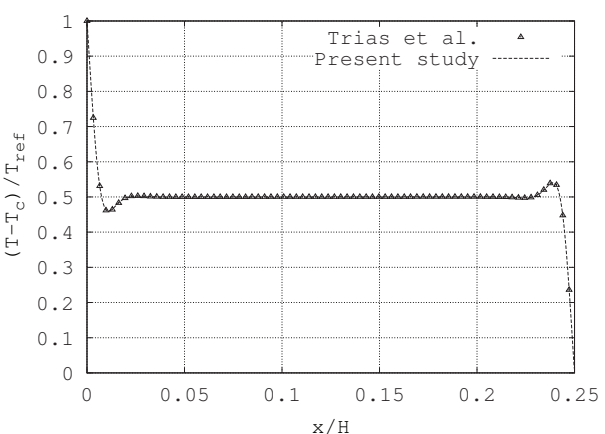

(a) Temperature at $y / H=0.5$

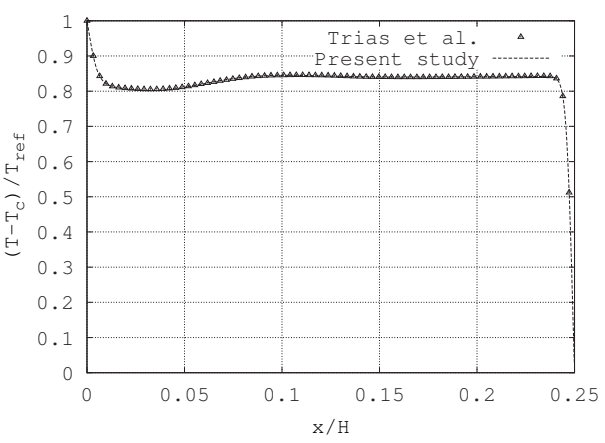

(c) Temperature at $y / H=0.9375$

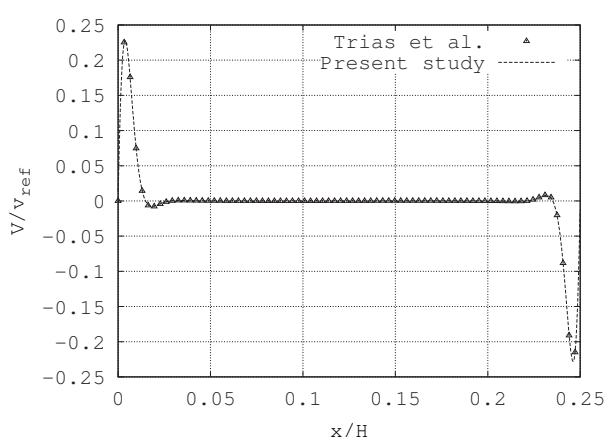

(b) Vertical velocity at $y / H=0.5$



(d) Vertical velocity at $y / H=0.9375$

Figure 3: Validation of the numerical model. Comparison with DNS results from [28].

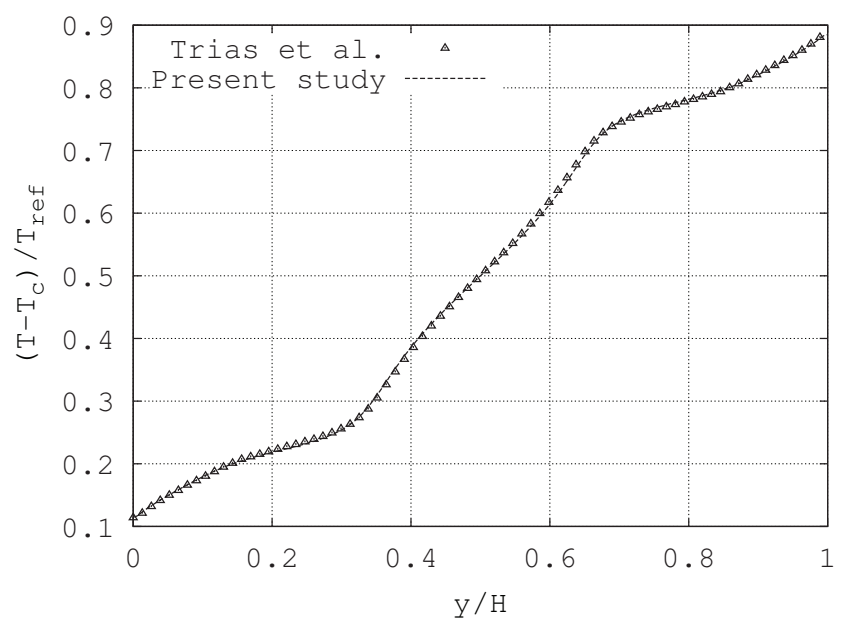

Figure 4: Temperature profile at $x / H=0.125$. Comparison with DNS results from [28]. 
In fact, as can be observed in Figure 4, the temperature profile along the cavity height is well represented, which implies a good prediction of the stratification, transition to turbulence location, and Nusselt number, among other features of this complex flow.

\section{Numerical results}

\subsection{Mesh refinement studies}

In order to capture all features of the flow, an adequate spatial discretization has to be performed. For the studied flow, viscous and thermal boundary layers at the isothermal confining walls are expected to dictate the smallest scales, while the grid size at the bulk zone must be smaller than the smallest scales. An a priori estimate of the boundary layer has shown that for the studied mean Prandtl number, the thermal boundary layer is thinner than the viscous one as $\delta_{t} \sim H / R a^{0.25}$ and $\delta_{\nu} \sim \operatorname{Pr}^{0.5} \delta_{t}$ [12]. Four different meshes are generated attaining the aforementioned criteria to carry out extensive mesh refinement studies, being the finest mesh of 864075 CVs $(615 \times$ 1405). All the meshes guarantee sufficient CVs within the boundary layers. Details of the tested meshes are given in Table 2. Although not shown here for the sake of brevity, the main parameters of the flow are compared in detail for the tested meshes. Comparisons have shown good agreement for meshes $I I I$ and $I V$, resulting in, for instance, relative differences of as low as $1.8 \%$ and $2.1 \%$ for time-averaged Nusselt number and time-averaged wall shear stress respeectively. Considering these two meshes, first-order statistics are in fair good agreement, even though mesh $I I I$ is more than 3 times coarser than mesh $I V$. 


\begin{tabular}{rcccc}
\hline Mesh & $N_{X}$ & $N_{Y}$ & $\Delta x_{\min }$ & $\Delta y_{\min }$ \\
\hline I & 177 & 913 & $1.36 \times 10^{-4}$ & $1.09 \times 10^{-3}$ \\
II & 227 & 913 & $1.36 \times 10^{-4}$ & $1.09 \times 10^{-3}$ \\
III & 227 & 1221 & $1.36 \times 10^{-4}$ & $8.19 \times 10^{-4}$ \\
IV & 615 & 1405 & $5.44 \times 10^{-5}$ & $7.12 \times 10^{-4}$ \\
\hline
\end{tabular}

Table 2: Main parameters of the meshes used in the mesh resolution studies. $N_{X}$ and $N_{y}$ are the number of CVs; $\Delta x_{\min }$ and $\Delta y_{\min }$ are the smallest wall-normal distances in horizontal and vertical directions, respectively.

In order to further assess the quality of the mesh resolution used in the present computations, an a posteriori analysis comparing the actual mesh size $\mathrm{h}\left(h \equiv(\Delta x \times \Delta y)^{1 / 2}\right)$ with the theoretical smallest scales of the flow is carried out.



Figure 5: Ratio of $h / L_{d}$ along vertical lines $\mathrm{x}=\mathrm{H} / 4 \Gamma, \mathrm{H} / 2 \Gamma, 3 \mathrm{H} / 4 \Gamma$.

In figure 5 , the profile of the ratio $h / L_{d}$ over the cavity height at three different positions $(x / H=1 / 4 \Gamma, 1 / 2 \Gamma, 3 / 4 \Gamma)$ is given, where $L_{d}=\nu^{1 / 2} / \hat{\eta}^{1 / 6}$ is the enstrophy dissipation length scale, and $\hat{\eta}$ is the enstrophy dissipation rate. The vertical profiles are chosen so as to have representative values 
for the whole cavity, and the positions are selected at the midwidth and in the vicinity of the hot and cold isothermal walls to account for different flow regimes in the cavity. As can be observed in the figure, for the finest computational mesh (mesh $I V$ ) the grid size is of 1 to 2 orders of magnitude smaller than the reference length scale, which shows that the selected grid is more than adequate to solve the smallest scales of the flow.

\subsection{Instantaneous flow}

Owing to results for similar flow parameters reported by several authors during the last decades (see for example $[2,3,6,7,8,28]$ ), a complex flow with laminar and turbulent regions is expected. An additional difficulty is the relatively large value of $\Gamma$ which, in case of a possible early transition to chaotic state in one (or both) of the vertical boundary layers, can make both to interact, resulting in a rather complicated flow.

In order to analyse the dynamics of the flow, it can be useful to plot the transient evolution of the temperature at a given probe location within the domain. In Figure 6(a), the temperature at the center of the cavity is depicted for the OB solution (BSQ10) and two of the NOB solutions (NOB10 and NOB60). The mean temperatures at the center of the cavity for both NOB cases are lower than that of the OB case, being the temperature for the NOB60 the lowest one. Moreover, the temperature fluctuations are of different intensity for all the cases. In Figure 6(b), accumulated temperature fluctuations are plotted from $t / t_{r e f}=300$. For the $B S Q 10$ case, the temperature fluctuations are quite low, indicating a quasi-laminar flow at the center of the cavity. However, for the NOB60 case, the flow is turbulent with higher fluctuations. Note that for this case, the average fluctuations are of about 5 


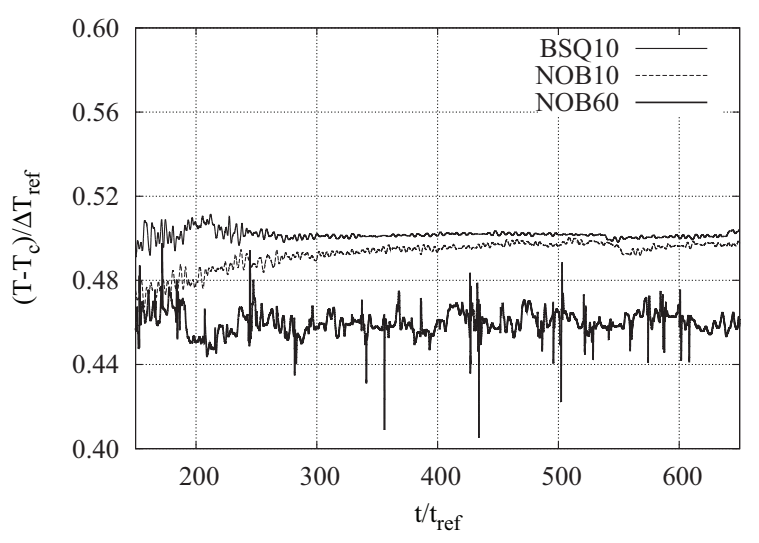

(a)

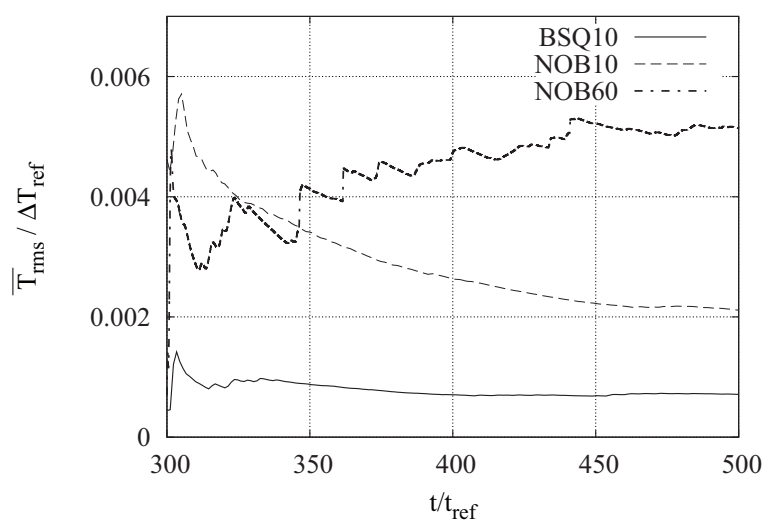

(b)

Figure 6: (a) Temperature evolution at the center of the cavity, (b) average accumulated temperature fluctuation; for (from bottom to top) BSQ10, NOB10, and NOB60 cases.

times higher with respect to the $B S Q 10$ case. From a qualitative analysis of the time dependent cavity center temperature and fluctuations, significant NOB effects can be anticipated.

Instantaneous isotherms can give more information about the behaviour of the flow. In Figure 7, the instantaneous temperature for the studied cases are given at $t / t_{\text {ref }}=700$ by means of 28 homogenously spaced isother- 


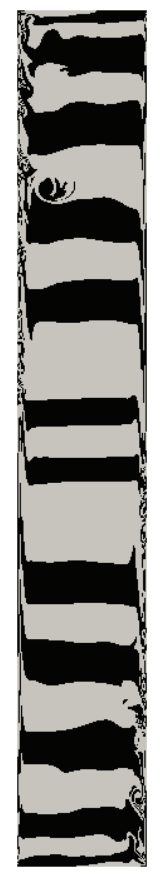

(a)

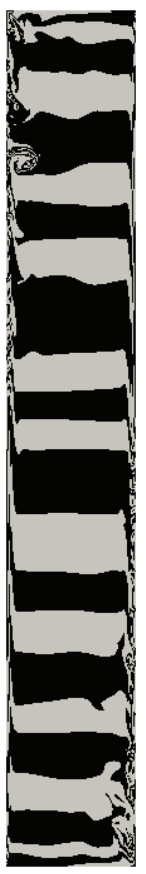

(b)

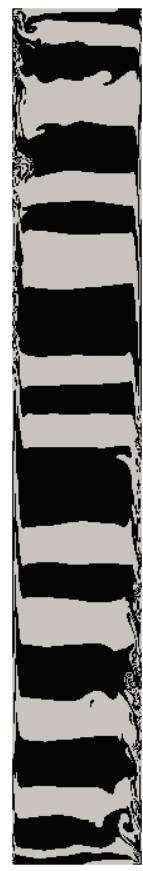

(c)

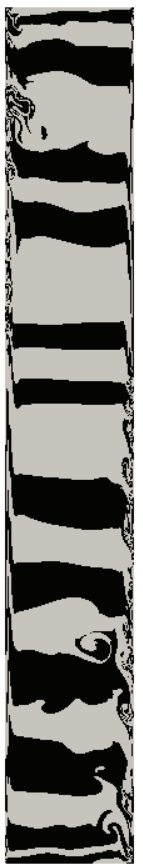

(d)

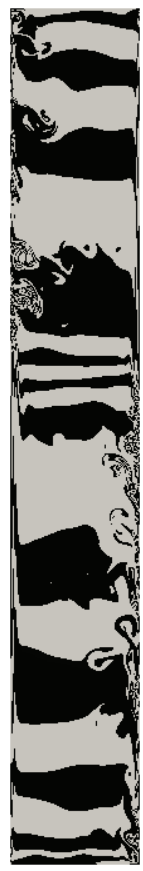

(e)

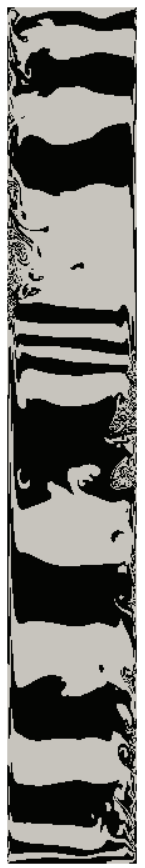

(f)

Figure 7: Instantaneous temperature isotherms at $t / t_{\text {ref }}=700$ for (a) BSQ10, (b) NOB10, (c) NOB20, (d) NOB30, (e) NOB40, and (f) NOB60.

mal contour lines between $\Phi\left(\Phi=\left(T-T_{c}\right) /\left(T_{h}-T c\right)\right)=0$ and $\Phi=1$, creating 27 regions in the form of zebra patterns. In all the flows, quasihorizontal isothermal contour lines are formed except for the regions in the vicinity of the vertical boundary layers, where higher temperature gradients are observed. Transition phenomenon can be qualitatively caught at some stage downstream on the hot and cold horizontal walls, while the position where this takes place changes significantly depending on the case. In cases BSQ10, NOB10, NOB20, and NOB30 (see Figures 7(a)-7(d)), tiny instabilities around midheight of the cavity (hot wall) can be noticed, however 
separation takes place about one fourth cavity height downstream. Similar behaviour can be perceived at the cold wall. As for the NOB40 and NOB60 cases (see Figures 7(e)-7(f)), the physics of the flow is different. In these flows, instead of the preliminar weak instabilities followed by the transition at somewhere downstream of the flow, the intensified instabilities accomplish transition to turbulence, injecting large eddies to the core of the cavity. These two flows show a more chaotic behaviour.

The OB solution is symmetric around the center of the cavity as can be seen in Figure 7(a), where isotherms are placed symmetrically around the central isotherm. For the NOB10 case (see Figure $7(\mathrm{~b})$ ), the symmetry is slighty broken, as the warmest (top of the cavity) and the coldest (bottom of the cavity) regions are located in a similar manner to those of the OB solution. The deviation from the OB solution can be observed specially at the core of the cavity, where some regions extend over the colder neighbouring region thus breaking the symmetry. Figures $7(\mathrm{c})$ and $7(\mathrm{~d})$ show a similar trend for the NOB20 and NOB30 cases, although for the NOB30 case, the discrepancies with respect to the $\mathrm{OB}$ solution are more pronounced. NOB effects are dominant in the NOB40 and NOB60 cases (Figures $7(\mathrm{e})-7(\mathrm{f})$ ). Symmetry no longer exists in these flows. In the NOB 40 case, the region containing the mean temperature of the cavity $\left(T_{m}\right)$ shifts upwards, resulting in colder cavity center. In both the hot and cold walls the instabilities move slightly downstream and get intensified. In between the transition locations of the hot and cold walls, 6 regions of isotherms shrink to form a highly stratified zone. This zone is bounded from the top and bottom by a large region of isotherm flow, which is formed as a consequence of enhanced horizontal 
motion. Similar pronounced NOB effects are observed in the NOB60 case. With respect to the previous NOB4O case, the transition in the cold wall occurs upstream, thus enhancing the active mixing in the geometric center of the cavity. In the hot wall, the transition point occurs slightly downstream. An upward shift in the highly stratified zone is observed with respect to the NOB 40 , due to upward shifts in the transition to turbulence locations of the both hot and cold boundary layers.

The NOB effects can be attributed to the behaviour of the vertical boundary layers. So as to contrast the initial ideas on the physics of the flow, the magnitude of the instantaneous velocity vector is plotted in Figure 8.

According to Figure 8, qualitatively speaking, OB solution does not differ from the NOB10, NOB20, and NOB30 significantly. In the hot wall, initial weak instabilities are followed by the transition to turbulence before the flow reaches the horizontal confining wall. Similar situation holds for the cold wall, where the transition to turbulence location comes slightly upstream. As a consequence, the turbulent fluctuating zone is extended, while the intensity of these fluctuations is reduced with respect to the hot wall region. As for the NOB 40 case, the physics of the flow changes due to strong instabilities in the hot wall, which results in a more pronounced boundary layer separation. The instabilities in the cold wall are also magnified, but less than those in the hot wall. As it comes to the NOB60 case, the same trend continues, being the flow structure similar to the NOB4O case. Transition to turbulence occurs slightly downstream (upstream) in the hot (cold) wall.

Instantaneous flow parameters can help to understand the physics of the flow. However to reach confident conclusive ideas, it is necessary to consider 


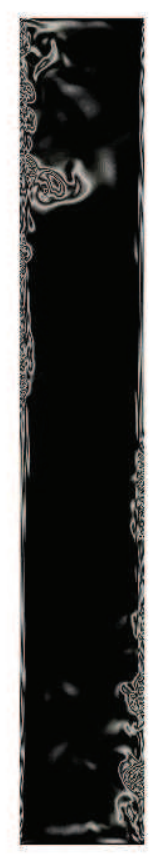

(a)

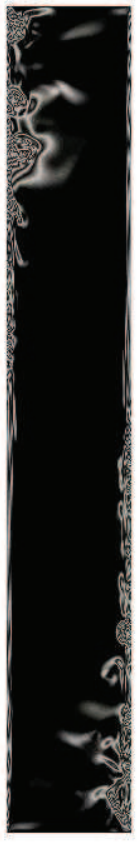

(b)

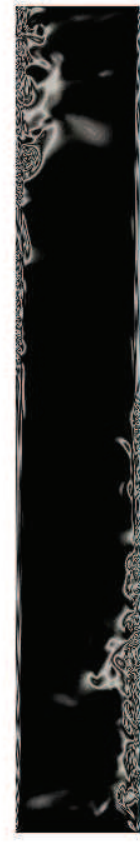

(c)

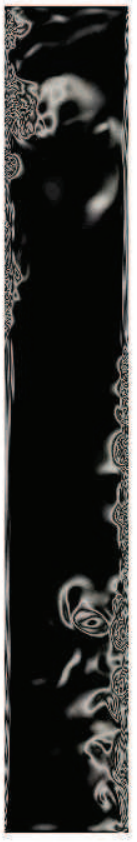

(d)
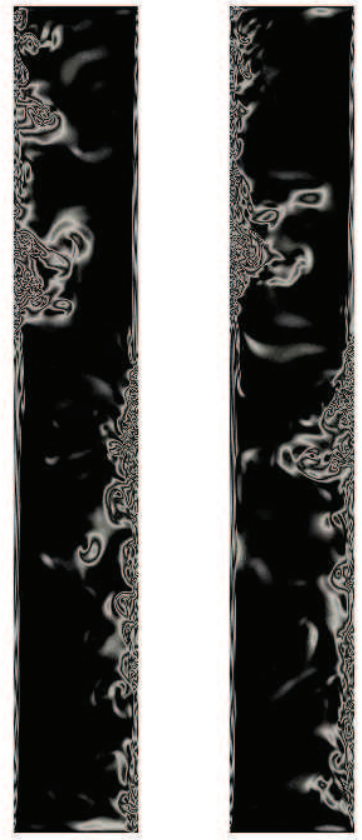

(e)

(f)

Figure 8: Instantaneous magnitude of the velocity vector at $t / t_{r e f}=700$ for (a) $B S Q 10$, (b) NOB10, (c) NOB20, (d) NOB30, (e) NOB40, and (f) NOB60.

the mean flow parameters. Thus, in the next section, the first order statistics are considered.

\subsection{Mean flow parameters}

As the flow under consideration is complex with time scales of different orders of magnitude, it is imperative to analyse the flow for adequate time integration. In order to assure that time-independent solution is obtained, numerical probes are placed in key locations representative of flows with different regimes. 


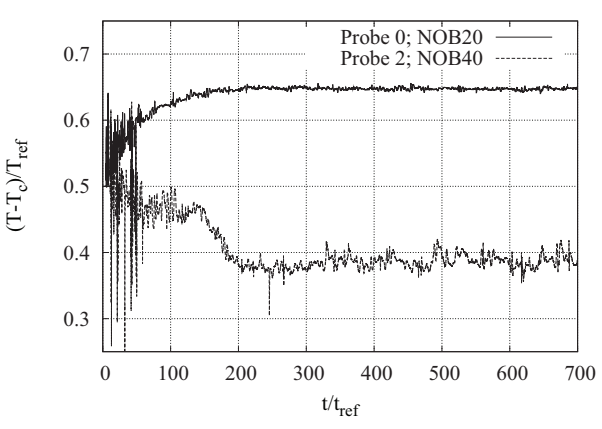

(a)

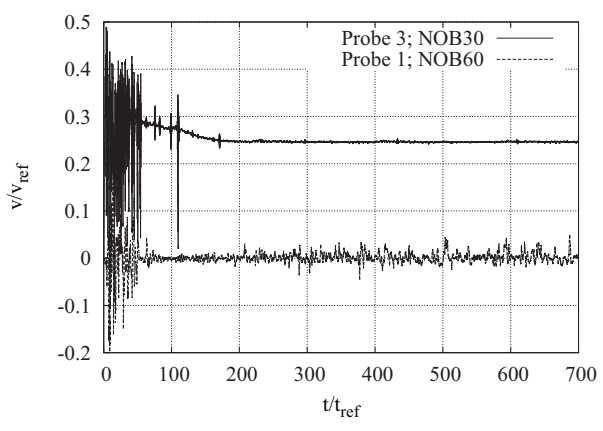

(b)

Figure 9: (a) Transient temperature and (b) vertical velocity at different probe positions and cases. Probe $0(1 / 2 \Gamma, 0.9)$; Probe 1 (1/2Г, 0.7$)$; Probe $2(1 / 2 \Gamma, 0.5)$; Probe $3(0.002,0.5)$.

In Figure 9, the time evolution of temperature and vertical velocity component are plotted at different probe locations. The positions of these probes are: Probe 0 at $(x / H, y / H) \equiv(1 / 2 \Gamma, 0.9)$, Probe 1 at $(1 / 2 \Gamma, 0.7)$, Probe 2 at $(1 / 2 \Gamma, 0.5)$, and Probe 3 at $(0.002,0.5)$. In the figure, NOB20, NOB30, NOB40, and NOB60 variables are plotted together, so as to point out the different time scales of the flows at these locations. Although not shown here for the sake of brevity, 19 probes are placed in representative locations to check the corresponding time scales of the flow. In the light of the gathered information from all the probes, time integration started from $t / t_{r e f}=300$. In order to have converged statistics, the flow has then been integrated over a period of 400 time units.

In Table 3, the maximum and minimum vertical velocities reached in the boundary layer for each of the studied cases are summarized together with their locations. Vertical velocity component is chosen since it is dominant due to the tall cavity configuration. Maximum vertical velocity, $v_{\max }$, is observed in the vicinity of the hot wall, while the minimum vertical velocity, 


\begin{tabular}{ccccccc}
\hline & $v_{\max } / v_{\text {ref }}$ & $x_{\max }^{*}$ & $y_{\max }^{*}$ & $v_{\min } / v_{\text {ref }}$ & $x_{\min }^{*}$ & $y_{\min }^{*}$ \\
\hline BSQ10 & 0.245 & $1.87 \times 10^{-3}$ & $4.65 \times 10^{-1}$ & -0.245 & $1.87 \times 10^{-3}$ & $4.65 \times 10^{-1}$ \\
NOB10 & 0.247 & $1.80 \times 10^{-3}$ & $4.91 \times 10^{-1}$ & -0.241 & $1.94 \times 10^{-3}$ & $4.37 \times 10^{-1}$ \\
NOB20 & 0.251 & $1.80 \times 10^{-3}$ & $5.13 \times 10^{-1}$ & -0.236 & $1.94 \times 10^{-3}$ & $4.12 \times 10^{-1}$ \\
NOB30 & 0.254 & $1.72 \times 10^{-3}$ & $5.37 \times 10^{-1}$ & -0.234 & $2.02 \times 10^{-3}$ & $3.87 \times 10^{-1}$ \\
NOB40 & 0.297 & $1.72 \times 10^{-3}$ & $5.30 \times 10^{-1}$ & -0.260 & $2.17 \times 10^{-3}$ & $3.91 \times 10^{-1}$ \\
NOB60 & 0.308 & $1.65 \times 10^{-3}$ & $5.60 \times 10^{-1}$ & -0.254 & $2.24 \times 10^{-3}$ & $3.62 \times 10^{-1}$ \\
\hline
\end{tabular}

Table 3: Maximum and minimum vertical velocities, $v_{\max } / v_{\text {ref }}$ and $v_{\min } / v_{\text {ref }}$ respectively, and their locations. $x_{\max }^{*}$, and $x_{\min }^{*}$ are the normal distances from hot and cold wall respectively. $y_{\max }^{*}$ and $y_{\min }^{*}$ are the vertical distance from the leading edge, i.e. from the bottom and the top of the cavity respectively. 
$v_{\text {min }}$, is registered in the vicinity of the cold wall. For a direct comparison of the cases, the locations are expressed in terms of the wall normal distances to the corresponding isothermal walls and vertical distances from the upstream adiabatic horizontal wall (see the caption of Table 3). Note that for $B S Q 10$, the maximum and minimum velocities are equal in absolute value, being symmetric the locations where these extrema are encountered. For the NOB cases, neither the absolute magnitudes nor their locations are symmetric and, in the hot wall the maximum values increase slightly (within 4 $\%$ with respect to $B S Q 10)$ up to NOB 40 case, where a big deviation (approximately $21 \%$ ) takes place. In accordance with the observations of the previous section regarding isotherm contour lines, with the NOB40 case the configuration of flow undergoes a significant change, which is also reflected in the vertical velocity peaks in the Table 3. However, when the temperature difference is further increased (i.e. NOB60) the structure of the flow does not differ essentially from the NOB40 case. The location where the vertical maximum (minimum) occurs in the hot (cold) wall goes downstream (upstream). The absolute value of the maximum (minimum) velocity increases (diminishes) gradually as the temperature difference increases until the NOB 40 case, where there is an abrupt increase. Then, a gradual increase (decrease) in the velocity peaks occurs as the temperature difference further rises by $20 \mathrm{~K}$. In all NOB cases, the velocities in the vicinity of the cold wall are smaller in absolute terms than their counterparts in the vicinity of the hot wall, indicating thicker boundary layer. Note that the relative change in the vertical velocity (i.e. $\frac{\left|v_{\max }\right|-\left|v_{\min }\right|}{\left|v_{\max }\right|}$ ) increases with the temperature difference. In Figure 10, vertical velocity profiles in the vicinity of the hot wall are 


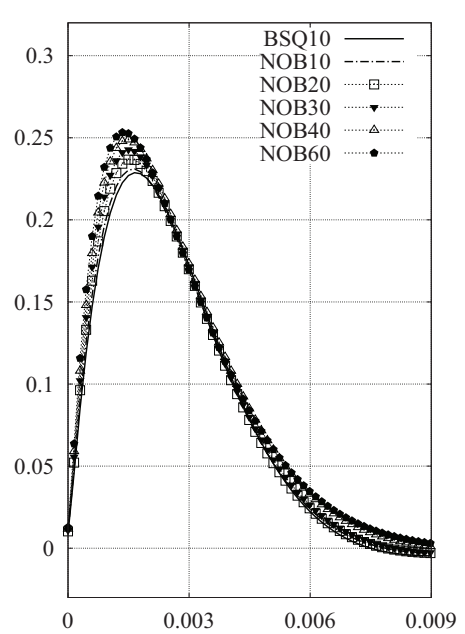

(a)

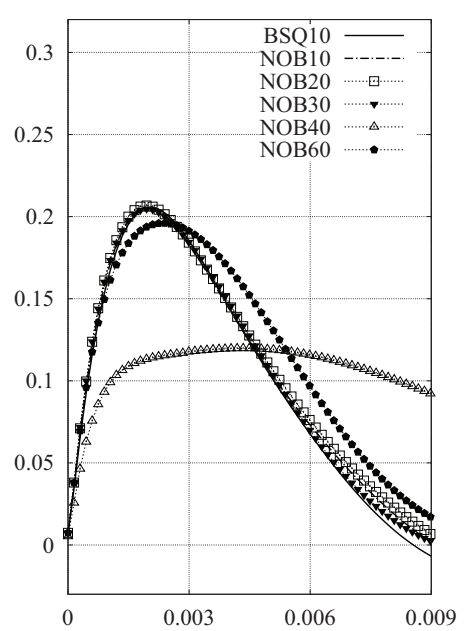

(c)

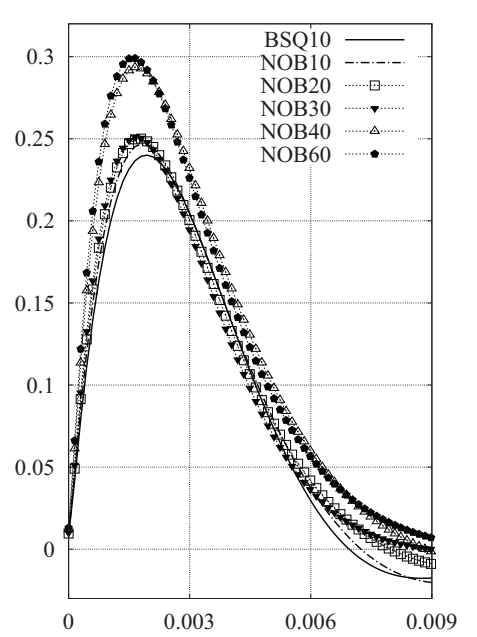

(b)

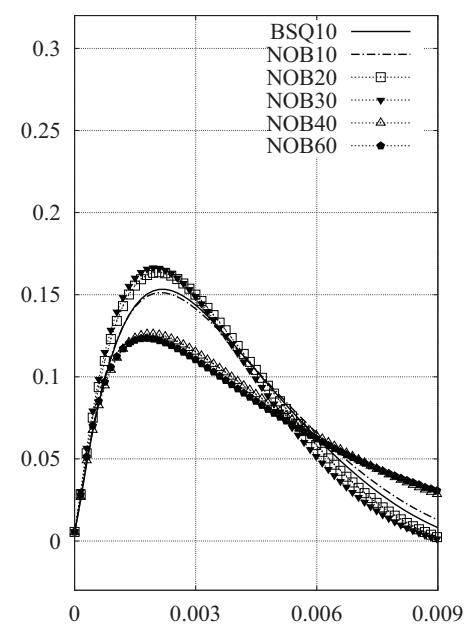

(d)

Figure 10: Vertical velocity profile $v / v_{\text {ref }}$ in the vicinity of the hot wall at different locations. X-axis corresponds to normal distance to the hot wall. (a) $\mathrm{y} / \mathrm{H}=0.3$ (b) $\mathrm{y} / \mathrm{H}=0.5$ (c) $\mathrm{y} / \mathrm{H}=0.65$ (d) $\mathrm{y} / \mathrm{H}=0.8$.

plotted for different cavity heights $(y / H=0.3,0.5,0.65,0.8)$. Note that only a wall-normal distance of approximately $6 \%$ of the cavity width is represented in the plots in order to highlight the boundary layer behaviour. In 
Figure $10($ a) vertical velocity at $y / H=0.3$ is given. At this location, the maximum velocity slightly increases with the temperature difference. However, at the midheight of the cavity the discrepancies between the cases get more pronounced (see Figure 10(b)). At this location, NOB10, NOB20, and NOB30 cases behave very similary, while for the two highest temperature difference cases, a significant departure from the OB solution is observed. This is in agreement with the data presented in Table 3. At $y / H=0.65$ (see Figure 10(c)) the BSQ10, NOB10, NOB20, and NOB30 cases have indistinguishable behaviour. In this location the boundary layer gets thicker and the velocity reduces with respect to the previous location. As for the NOB40 case, a major desceleration is observed. The vertical velocity component reduces more than $50 \%$ due to the flow separation taking place, while for the NOB60 case, the transition takes place slightly downstream, which justifies a boundary layer thickness and vertical velocity magnitude similar to the three lowest temperature difference solutions. Finally, at $y / H=0.8$, the flow gets more chaotic thus registering smaller vertical velocity components. This is especially true for the NOB40 and NOB60 cases where the vertical velocity is approximately $15 \%$ lower than the $B S Q 10$ solution. At the top of the cavity, BSQ10 and NOB10 cases behave identically while for temperature difference of 20 and $30 \mathrm{~K}$, the velocities are slightly higher.

In Figure 11, vertical velocity in the cold wall is plotted. For comparison purposes, these locations are equivalent to those selected in the hot wall. The loss of symmetry occurs as early as $y / H=0.7$ (see Figure 11(a)). At midheight of the cavity (see Figure 11(b)), the behavior of the NOB40 and NOB60 cases are totally different than the other cases, as the transition takes 


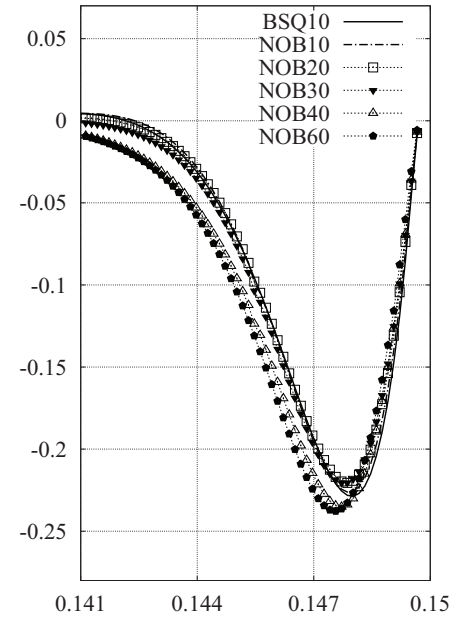

(a)

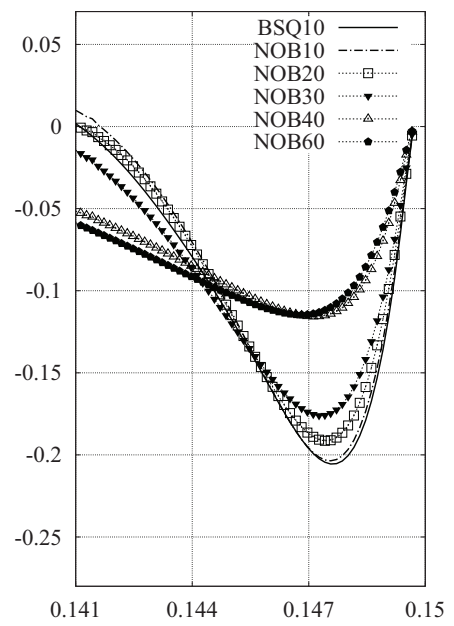

(c)

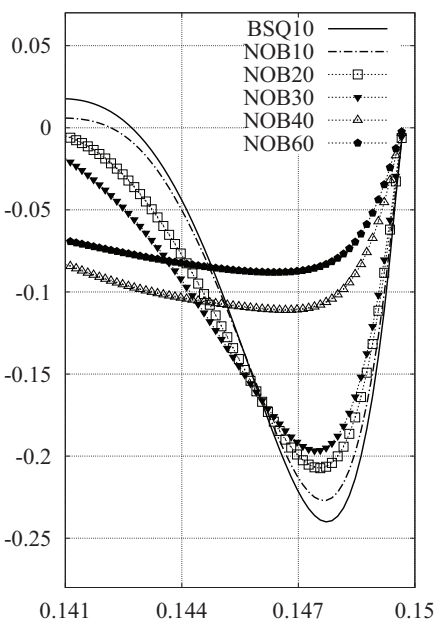

(b)

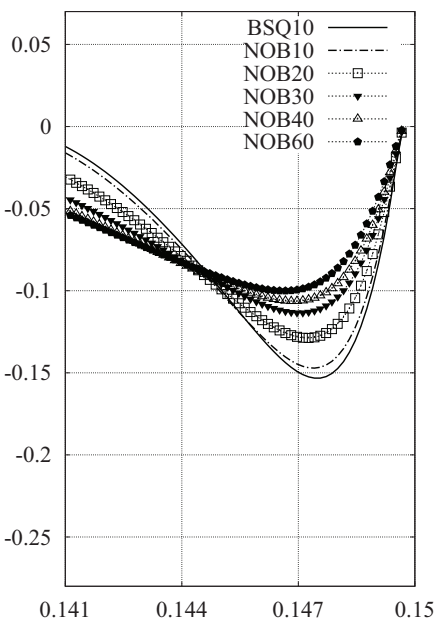

(d)

Figure 11: Vertical velocity profile $v / v_{\text {ref }}$ in the vicinity of the cold wall at different locations. $\mathrm{X}$-axis corresponds to normal distance to the hot wall. (a) $\mathrm{y} / \mathrm{H}=0.7$ (b) $\mathrm{y} / \mathrm{H}=0.5$ (c) $\mathrm{y} / \mathrm{H}=0.35$ (d) $\mathrm{y} / \mathrm{H}=0.2$.

place earlier than in hot wall. This can also be anticipated in Table 3, where in the cold wall the absolute maximum values are reached before midheight. As the flow goes downstream, similar effects as in the hot wall are observed, 
although the NOB effects break totally the symmetry, and the boundary layer gets significantly thicker than in hot wall (see Figures 11(c) and 11(d)). Note that concerning the flow separation, for the two highest temperature differences, the flow behaves differently in the vertical boundary layers. While in the hot wall the NOB4O transitions earlier, in the cold wall, in turn, instabilities occur earlier for the NOB60 case.

\begin{tabular}{llrr}
\hline & $N u$ & $y_{t r}^{\text {hot }} / H$ & $y_{t r}^{\text {cold }} / H$ \\
\hline BSQ10 & 231.18 & 0.56 & 0.56 \\
NOB10 & 231.85 & 0.59 & 0.53 \\
NOB20 & 232.23 & 0.61 & 0.50 \\
NOB30 & 233.92 & 0.64 & 0.48 \\
NOB40 & 251.07 & 0.66 & 0.50 \\
NOB60 & 248.85 & 0.69 & 0.47 \\
\hline
\end{tabular}

Table 4: Average Nusselt numbers and transition point at hot and cold wall. $y_{t r}^{\text {hot }} / H$ and $y_{t r}^{c o l d} / H$ are the vertical distance from the bottom and the top of the cavity, respectively.

Average Nusselt number is computed as $N u=\left.\overline{k \partial T / \partial n}\right|_{\text {wall }}$, where the subscript wall represents the location where the derivative is evaluated (i.e. hot or cold wall) and the overbar represents average over time. In Table 4, a summary of the computed values of the Nusselt number are given together with the location where transition occurs in hot and cold walls. For all cases, the location of the transition is defined as the position where $\overline{N u_{r m s}}$ peaks. In order to clarify the adopted criterion, the $\overline{N u_{r m s}}$ distribution in the hot and cold walls are plotted in Figure 12. Note that the figure is not to scale, and the graphs are shifted in y-direction to expose the transition to turbu- 
lence locations. However, the relative intensities of each peak value with respect to their upstream quasi-constant values are scaled. From the figure, the difference in physics of the flows can be appreciated. For the NOB40 and NOB60 cases, there is one large peak value unlike the lower temperature difference cases, which have a secondary broadband peak occuring downstream before reaching the horizontal boundary. In Table 4, the presented transition locations are consistent with the selected criterion. It should be pointed out that for the NOB40 and NOB60 cases, a small plateau prior to the $\overline{N u_{r m s}}$ peak is observed. The general trend in these two cases is different than for the lower temperature difference cases. This also supports the hypothesis of a change in the physics with the increase of temperature difference and the enhancement of the turbulent transport. In fact, in these two cases, transition to turbulence starts to occur slightly upstream the location where $\overline{N u_{r m s}}$ peaks.

Regarding the magnitude of the average Nusselt number, significant differences are not observed up to a temperature difference of $40 \mathrm{~K}$. Note that the increment in the average Nusselt number is only about $1 \%$ (for temperature differences of 10, 20, and $30 \mathrm{~K}$ ). However, considering the location of transition to turbulence, the loss of symmetry is certified in all cases.

In order to highlight the NOB effects, local average Nusselt number in the early transitioning cold wall is plotted in Figure 13. The observed similarities in the physics of the flow indicates similar average Nusselt numbers for NOB10, NOB20, and NOB30 cases, and the minor increment with temperature difference is due to upstream transition in the cold wall. As for 40 and $60 \mathrm{~K}$, the change in the physics of the flow can be confirmed in Figure 13(b). 


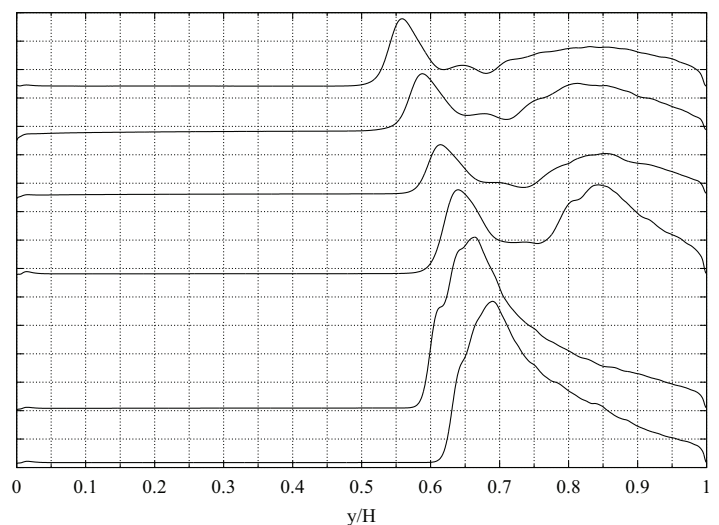

(a)

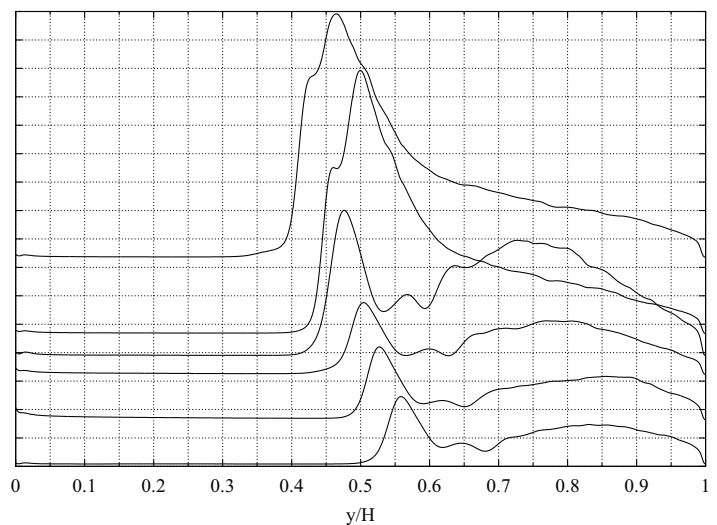

(b)

Figure 12: $\overline{N u_{r m s}}$ from the leading edge (a) in the hot wall (from top to bottom), (b) in the cold wall (from bottom to top); for BSQ10, NOB10, NOB20, NOB30, NOB40, NOB60, respectively.

The enhancement in heat transfer for these temperature differences cannot be explained only by an earlier transition in the cold wall. The higher peak in Nusselt number and its local values after transition indicate a more chaotic flow, resulting in up to $8 \%$ higher average Nusselt numbers.

Thermal behaviour within the cavity can be representatively analysed by means of the vertical profile in the half width $(x / H=1 / 2 \Gamma)$ location. 


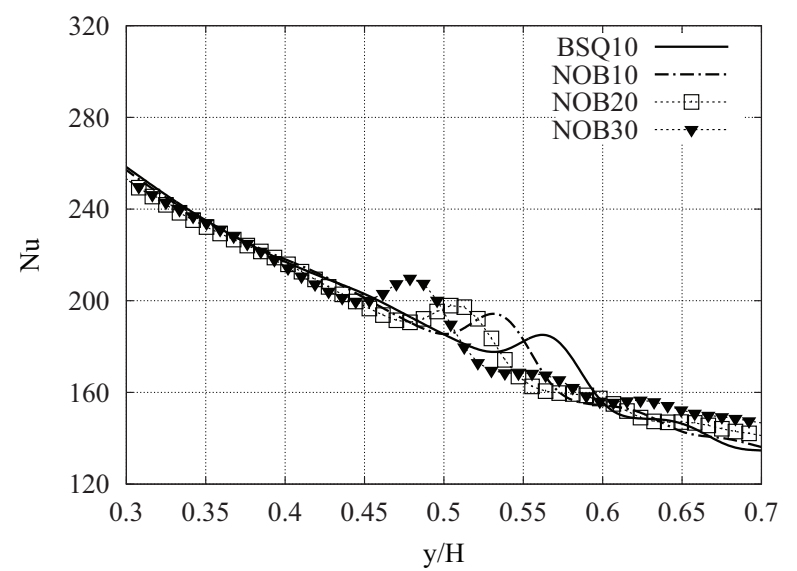

(a)

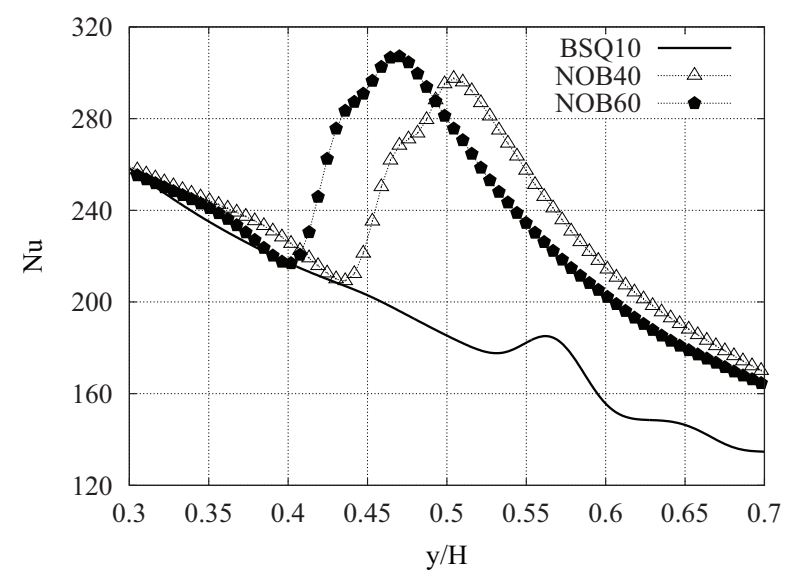

(b)

Figure 13: Local average Nusselt number in cold wall for temperature differences of (a) 10, 20, and $30 \mathrm{~K}$; (b) 10, 40, $60 \mathrm{~K}$.

In Figure 14, temperature profiles and the stratification number $(\partial T / \partial y)$ in this location are comparatively shown. The change in the physics of the flow can be clearly observed in Figure 14(b), where NOB40 and NOB60 cases are presented with the $B S Q 10$ case. It can be seen how OB solution presents lower temperatures in the bottom of the cavity. The stratification region occurs in the midheight of the cavity for the $B S Q 10$ case, being the 
stratification value $\partial T / \partial y=0.97$ at the center. The upward shift in the highly stratified zone for NOB40 and NOB60 cases can be observed in the same figure.

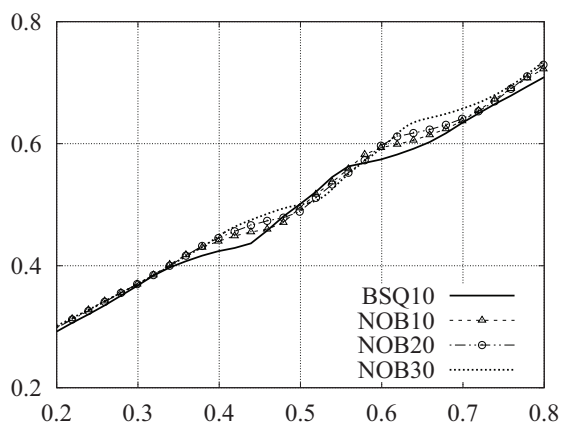

(a)

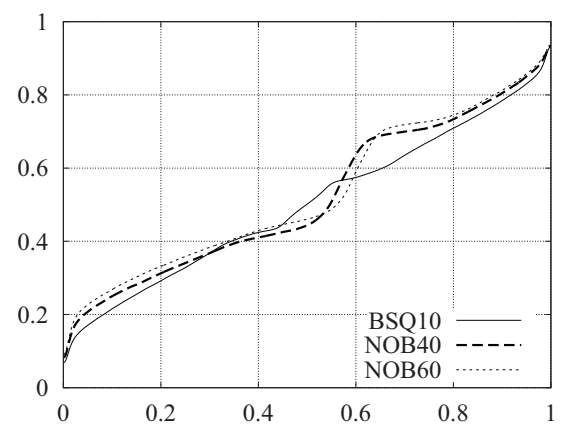

(b)

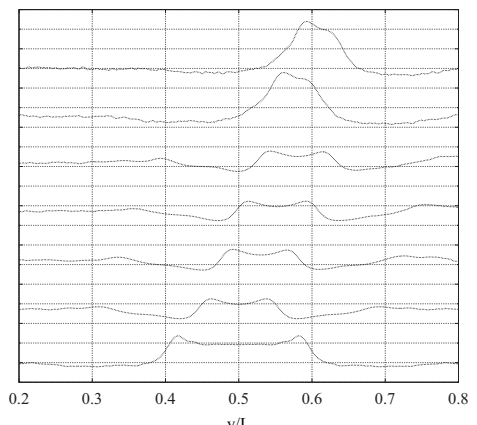

(c)

Figure 14: Comparative temperature and stratification profiles at $(x=1 / 2 \Gamma)$ location. (a) temperature for BSQ10, NOB10, NOB20, NOB30; (b) temperature for BSQ10, NOB40, NOB60. (c) stratification number from bottom to top for Trias et al. (2010), BSQ10, NOB10, NOB20, NOB30, NOB40, NOB60. Stratification plots are seperated by 3 vertical stratification units in order to emphasis the differences.

In Figure 14(a), NOB10, NOB20, NOB30 cases are compared with the $B S Q 10$ case. Note that in order to highlight the behaviour in the core of the cavity, the values within $0.2<y / H<0.8$ are only shown in this plot. The same trend observed for the $B S Q 10$ case is qualitatively reproduced by 
NOB10, NOB20, and NOB30 cases, while the highly stratified zone shifts slightly upwards as the temperature difference increases. This can be better observed in Figure 14(c) where the stratification number profiles of the tested cases are plotted. In order to emphasize the NOB effects, the plots are separated by three stratification number units. From bottom to top, $R a=10^{11}$ with aspect ratio of 4 for air by Trias et al.[11], BSQ10, NOB10, NOB20, NOB30, NOB40, and NOB60 are plotted, respectively. Note that the two bottommost cases are symmetric with respect to the cavity center as expected. In the literature, the stratification number is reported at the center of the cavity for DHC flows. While this approach can be acceptable for symmetric flows, it can be misleading for the NOB cases presented in this work. In symmetric flows, the core region with higher stratification number is located in the center of the cavity. However for NOB cases a shift in the location of this higher stratification zone is observed. In order to contrast the NOB effects, the higher stratification core zone is defined as the region between two local stratification peak locations. This is further remarked in Table 5.3, where the locations of the stratification peaks and their respective stratification numbers are given. $\Delta y_{\text {core }}$ stands for the size of the stratified core region (i.e. distance in between local peaks). Note that for the two higher temperature difference cases, the stratification number reaches significantly higher values. For these cases, $\Delta y_{\text {core }}$ is calculated using the minimum locations of the second derivative of temperature. This is due to the fact that in these flows, unlike the lower temperature difference cases, only one stratification peak is observed.

In Figure 15, the dimensionless time-averaged wall shear stress is pre- 


\begin{tabular}{cccc}
\hline CASE & $y_{p} / H$ & $\begin{array}{c}\text { peak stratification } \\
\text { number }\end{array}$ & $\Delta y_{\text {core }}$ \\
\hline BSQ10 & 0.46 & 1.25 & 0.08 \\
NOB10 & 0.49 & 1.27 & 0.08 \\
NOB20 & 0.51 & 1.22 & 0.08 \\
NOB30 & 0.54 & 1.29 & 0.07 \\
NOB40 & 0.56 & 2.79 & 0.02 \\
NOB60 & 0.59 & 2.90 & 0.02 \\
\hline
\end{tabular}

Table 5: Parameters of the stratification number for the studied cases.

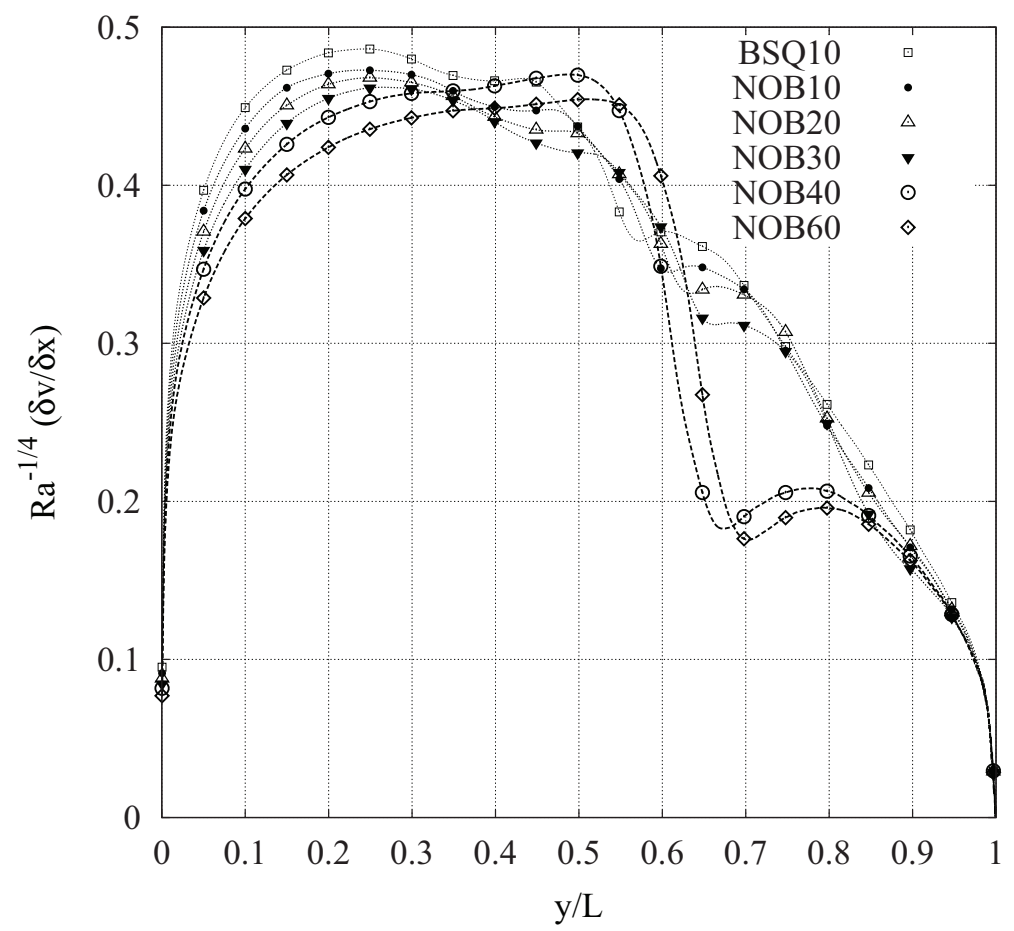

Figure 15: Dimensionless time-averaged wall shear stress at the hot wall scaled by $R a^{-1 / 4}$ for the tested cases. 
sented for the studied cases. In accordance with the previous indicators, the sudden decrease in the wall shear stress for the NOB40 and NOB60 cases certifies a remarkable thickening of the boundary layer. This is not observed in the lower temperature difference cases, where the reduction in the shear stress occurs in a more gradual manner. This is in agreement with the reduced horizontal eddy ejecting motion with respect to the $N O B 40$ and NOB60 cases.

\section{Conclusions}

The relevance of the NOB effects in a tall two-dimensional DHC cavity filled with water at $R a=2.1 \times 10^{11}, \operatorname{Pr}=3.27$, and $\Gamma=6.67$ has been submitted to investigation. A second-order symmetry-preserving formulation for solving the governing equations has been used. The numerical model has been validated by means of the comparison with direct numerical simulations from the literature for a differentially heated cavity of aspect ratio 4, reproducing accurately the complex physics present in such configuration. NOB efects have been studied up to a temperature difference of $60{ }^{\circ} \mathrm{C}$. Timeaveraged flow parameters and representative instantaneous fields have been presented. The numerical results reveal that significant discrepancies in the dynamics of the flow and heat transfer can be observed if the NOB effects are considered. Up to the temperature difference of $30{ }^{\circ} \mathrm{C}$, OB solution can estimate the heat transfer with about $1 \%$ error, although the loss of symmetry of the flow is certified even for a temperature difference of $10 \mathrm{~K}$. At the temperature difference of $40{ }^{\circ} \mathrm{C}$, the physics of the flow undergoes a major change, affecting transition position, heat transfer, and the stratification sub- 
stancially. The boundary layers at hot and cold isothermal confining walls behave differently. At this temperature difference, OB solution underestimates the heat transfer by about $8 \%$. OB solution predicts the transition to turbulence location symmetrically at $56 \%$ of the cavity height from the leading edge. However, when the NOB effects are considered, the transition location tends to move downstream in the hot wall, and upstream in the cold wall. Especially for temperature differences higher than $30^{\circ} \mathrm{C}$, the discrepancies are pronounced. In the case of the temperature difference of $40{ }^{\circ} \mathrm{C}$, the transition occurs at $66 \%$ and $50 \%$ of the cavity height in the hot and cold walls, respectively. Due to the upward shift in both boundary layers, the stratification region changes, resulting in higher stratification numbers. For these cases, the interaction between the two boundary layers results in a more complex flow, where neglecting NOB effects may lead to erroneous estimations of the phenomena taking place.

\section{Acknowledgements}

This work has been financially supported by the Ministerio de Educación

y Ciencia, Secretaría de Estado de Universidades e Investigación, Spain (Ref. ENE2009-09496) and by the Collaboration Project between Universitat Politècnica de Catalunya and Termo Fluids S.L.

\section{References}

[1] D. Gray, A. Giorgini, The validity of the Boussinesq approximation for liquids and gases, International Journal of Heat and Mass Transfer 19 (1976) 545-551. 
[2] G. De Vahl Davis, Natural Convection of Air in a Square Cavity: a Benchmark Numerical Solution, International Journal for Numerical Methods in Fluids 3 (1983) 249-264.

[3] R. Henkes, F. van Der Vlugt, C. Hoogendoorn, Natural convection flow in a square cavity calculated with low reynolds-number turbulence models, International Journal of Heat and Mass Transfer 34 (1991) 377-388.

[4] T. Fusegi, J. M. Hyun, K. Kuwahara, B. Farouk, A numerical study of three-dimensional natural convection in a differentially heated cubical enclosure, International Journal of Heat and Fluid Flow 34 (6) (1991) $1543-1557$.

[5] R. J. A. Janssen, R. A. W. M. Henkes, C. J. Hoogendorn, Transition to Time-Periodicity of a Natural-Convection Flow in a 3-D Differentially Heated Cavity, International Journal of Heat and Mass Transfer 36 (11) (1993) 2927-2940.

[6] S. Xin, P. L. Queré, Direct numerical simulations of two-dimensional chaotic natural convection in a differentially heated cavity of aspect ratio 4, Journal of Fluid Mechanics 304 (1995) 87-118.

[7] P. Quere, M. Behnia, From onset of unsteadiness to chaos in a differentially heated square cavity, Journal of Fluid Mechanics 359 (1998) $81-107$.

[8] D. G. Barhaghi, L. Davidson, Natural convection boundary layer in a 5:1 cavity, Physics of Fluids 19 (12) (2007) 125106-125106. 
[9] F. X. Trias, A. Gorobets, M. Soria, A. Oliva, Direct numerical simulation of a differentially heated cavity of aspect ratio 4 with Rayleigh numbers up to 1E11 Part II: Heat transfer and flow dynamics, International Journal of Heat and Mass Transfer 53 (1) (2010) 674-683.

[10] J. Salat, S. Xin, P. Joubert, A. Sergent, F. Penot, P. Le Quere, Experimental and numerical investigation of turbulent natural convection in large air-filled cavity, International Journal of Heat and Fluid Flow 25 (2004) 824-832.

[11] F. X. Trias, A. Gorobets, M. Soria, A. Oliva, Direct numerical simulation of a differentially heated cavity of aspect ratio 4 with Rayleigh numbers up to 1E11 Part I: Numerical methods and time-averaged flow, International Journal of Heat and Mass Transfer 53 (1) (2010) 665-673.

[12] J. Patterson, J. Imberger, Unsteady natural convection in a rectangular cavity, Journal of Fluid Mechanics 100 (1) (1980) 65-86.

[13] R. Janssen, S. Armfield, Stability properties of the vertical boundary layers in differentially heated cavities, International Journal of Heat and Fluid Flow 17 (6) (1996) 547-556.

[14] M. Farhangnia, S. Biringen, L. J. Peltier, Numerical simulation of twodimensional buoyancy-driven turbulence in a tall rectangular cavity, International Journal for Numerical Methods in Fluids 23 (1996) 13111326.

[15] S. Paolucci, D. Chenoweth, Transition to chaos in a differentially heated vertical cavity, Journal of Fluid Mechanics 201 (1989) 379-410. 
[16] S. Paolucci, Direct numerical simulation of two-dimensional turbulent natural convection in an enclosed cavity, Journal of Fluid Mechanics 215 (1990) 229-262.

[17] S. Suslov, S. Paolucci, Stability of natural convection flow in a tall vertical enclosure under non-Boussinesq conditions, International Journal of Heat and Mass Transfer 38 (12) (1995) 2143-2157.

[18] H. Mlaouah, T. Tsuji, Y. Nagano, A study of non-Boussinesq effect on transition of thermally induced flow in a square cavity, International Journal of Heat and Fluid Flow 18 (1) (1997) 100-106.

[19] S. Szewc, J. Pozorski, A. Taniere, Modeling of natural convection with Smoothed Particle Hydrodynamics: Non-Boussinesq formulation, International Journal of Heat and Mass Transfer 54 (2011) 4807-4816.

[20] F. Robinson, K. Chan, Non-Boussinesq simulations of Rayleigh-Benard convection in a perfect gas, Physics of Fluids 16 (5) (2004) 1321.

[21] S. Madruga, H. Riecke, Non-Boussinesq convection at low Prandtl numbers: Hexagons and spiral defect chaos, Physical Review E 75 (2007) 026210 .

[22] G. Ahlers, E. Brown, F. F. Araujo, D. Funfschilling, S. Grossmann, D. Lohse, Non-Oberbeck-Boussinesq effects in strongly turbulent Rayleigh-Bénard convection, Journal of Fluid Mechanics 569 (2006) 409-445. 
[23] K. Sugiyama, E. Calzavarini, S. Grossmann, D. Lohse, Non-OberbeckBoussinesq effects in two-dimensional Rayleigh-Bénard convection in glycerol, Europhysics Letters (EPL) 80 (3) (2007) 34002.

[24] G. Ahlers, E. Calzavarini, F. Araujo, D. Funfschilling, S. Grossmann, D. Lohse, K. Sugiyama, Non-Oberbeck-Boussinesq effects in turbulent thermal convection in ethane close to the critical point, Physical Review E 77 (4) (2008) 046302.

[25] J. Schmalzl, M. Breuer, U. Hansen, On the validity of two-dimensional numerical approaches to time-dependent thermal convection, Europhys. Lett. 67 (3) (2004) 390-396.

[26] E. DeLuca, J. Werne, R. Rosner, F. Cattaneo, Numerical simulations of soft and hard turbulence: preliminary results for two-dimensional convection, Phys. Rev. Lett. 64 (20) (1990) 2370-2373.

[27] K. Sugiyama, E. Calzavarini, S. Grossmann, D. Lohse, Flow organization in two-dimensional non-Oberbeck-Boussinesq Rayleigh-Bénard convection in water, Journal of Fluid Mechanics 637 (2009) 105.

[28] F. X. Trias, M. Soria, C. D. Pérez-Segarra, A. Oliva, Direct Numerical simulations of two and three dimensional turbulent natural convection flows in a differentialy heated cavity of aspect ratio 4, Journal of Fluid Mechanics 586 (2007) 259-293.

[29] R. W. C. P. Verstappen, A. E. P. Veldman, Symmetry-Preserving Discretization of Turbulent Flow, Journal of Computational Physics 187 (2003) 343-368. 
[30] F. X. Trias, O. Lehmkuhl, A self-adaptive strategy for the time integration of navier-stokes equations, Numerical Heat Transfer. Part B 60 (2) (2011) 116-134.

[31] I. Rodríguez, R. Borrell, O. Lehmkuhl, C. D. Pérez-Segarra, A. Oliva, Direct Numerical Simulation of the Flow Over a Sphere at Re $=3700$, Journal of Fluids Mechanics 679 (2011) 263-287.

[32] I. Rodríguez, O. Lehmkuhl, R. Borrell, C. D. Pérez-Segarra, A. Oliva, Low-frequency variations in the wake of a circular cylinder at $R e=3900$, in: Proceedings of the 13th European Turbulence Conference, 2011.

[33] D. Kizildag, J. Ventosa, I. Rodriguez, A. Oliva, Non-OberbeckBoussinesq natural convection in a tall differentially heated cavity, in: Proceedings of the Fifth European Conference on Computational Fluid Dynamics ECCOMAS CFD 2010, 2010, pp. 1-13.

[34] I. Rodríguez, O. Lehmkuhl, R. Borrell, C. D. Perez-Segarra, On DNS and LES of natural convection of wall-confined flows: Rayleigh-Benard convection, in: H. Kuerten, B. Geurts, V. Armenio, J. Fröhlich (Eds.), Direct and Large-Eddy Simulation VIII (ERCOFTAC Series), Springer, 2011, pp. 389-394.

[35] Y. Morinishi, T. Lund, O. Vasilyev, P. Moin, Fully conservative higher order finite difference schemes for incompressible flow, Journal of Computational Physics 143 (1) (1998) 90-124.

[36] F. Felten, T. Lund, Kinetic energy conservation issues associated with 
the collocated mesh scheme for incompressible flow, Journal of Computational Physics 215 (2) (2006) 465-484. 